\title{
Optimal Medicine Distribution by Using AHP
}

\author{
Xinli Zhou \\ School of North China Electric Power University, Baoding 071000, China \\ 1297682657@qq.com
}

Keywords: Analytic Hierarchy Process; Optimal Medicine; Distribution.

\begin{abstract}
When an epidemic disease break out, vaccines will be not enough at first, so we should take so many factors into consideration when distribute the medicines. In this paper, we take Ebola epidemic for example. To allocate the limited medicine reasonably to epidemic regions, we adopt the Analytic Hierarchy Process (AHP) to determine the scale of medicine distribution. We take 3 indicators into consideration, which include Severity of Epidemic, Expenditure for Public Health and The Number of Medical Staff, then we compute the proportion of medicine distribution for three countries: Guinea, Liberia and Sierra Leone, finally, we distribute medicines according to the weight of the three countries correspondingly.
\end{abstract}

\section{Introduction}

It is no doubt that the Ebola epidemic has been the most serious global disease since March 2014. In October 8, 2014, the world medical association has announced that the world needed to realize that Ebola was a global crisis and not simply a problem for West Africa. As an increasing number of Ebola infections, a highly discussed topic is what we can do to control the crisis. If there is no effective drug control measures and the epidemic will continue to spread it, and spread through out of the world. Recently, a new medication can stop Ebola and cure patients whose disease is not advanced is invented while it is in small quantity. Due to the severe situation, we adopt the Analytic Hierarchy Process (AHP) to determine the scale of medicine distribution to optimize the epidemic's current strain.

\section{Analytic Hierarchy Process[1][2](AHP)}

Considering the scarcity of the medicine, reasonable distribution of drugs appears to be especially necessary. We adopt AHP to give weight to the Guinea, Liberia and Sierra Leone and obtain the amount of medicine needed respectively.

\section{The three-hierarchy structure}

Taking economic situation and severity of Ebola into consideration, we use 3 indexes to determine the weight of the three countries: Severity of epidemic, Per capital health expenditure and Medical staff per 10000 people. We construct the three hierarchy structure, which is shown in the following figure.

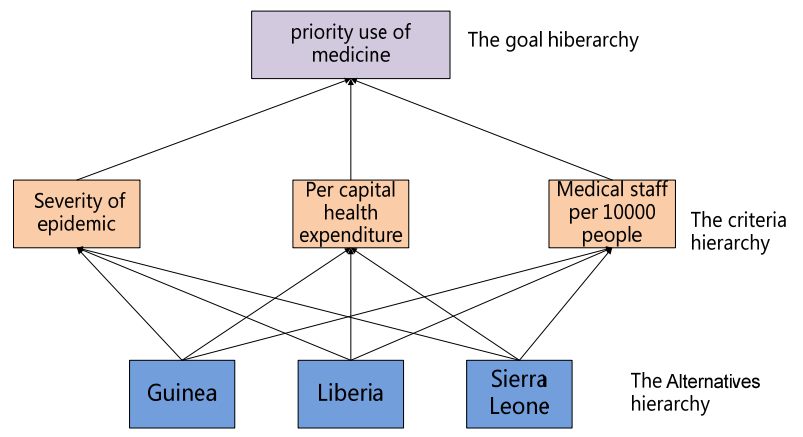

Fig. 1 The three hierarchy structure of model 


\section{Obtain the index weight:}

Establish pairwise comparison matrix.

Establish pairwise comparison matrix $A=\left(a_{i j}\right)$. Each entry ${ }^{a_{j k}}$ of the matrix represents the importance of the $j^{\text {th }}$ criterion relative to the $k^{\text {th }}$ criterion.

Where: If $a_{j k}>1$, then the $j^{\text {th }}$ criterion is more important than the $k^{\text {th }}$ criterion

If $a_{j k}<1$, then the $j^{\text {th }}$ criterion is less important than the $k^{\text {th }}$ criterion.

If two criteria have the same importance, then the entry $a_{j k}$ is 1 .

The entries $a_{j k}$ and $a_{k j}$ satisfy the following constraint:

$$
a_{j k} \times a_{k j}=1
$$

Obviously, $a_{j j}=1$ for all $j$. The relative importance between two criteria is measured according to a numerical scale from 1 to 9 , as shown in Table 1 :

Table 1 Table of relative scores

\begin{tabular}{ll}
\hline Value of $a_{j k}$ & Meaning \\
\hline 1 & $j$ and $k$ are equally important \\
3 & $j$ is slightly more important than $k$ \\
5 & $j$ is more important than $k$ \\
7 & $j$ is strongly more important than $k$ \\
9 & $j$ is absolutely more important than $k$ \\
$2,4,6,8$ & Intermediate values to reflect fuzzy inputs \\
\hline
\end{tabular}

Calculate the eigenvalues and eigenvector

The greatest eigenvalue of matrix $A$ is $\lambda$ and the corresponding eigenvector is $\omega$ :

$A \omega=\lambda \omega$

Where $A$ is the comparison matrix;

Do the consistency check

The consistence index (CI) and consistence radio (CR) can be calculated by formula (3)

$C I=\frac{\lambda-n}{n-1}$

Where: $n$ denotes the exponent number of matrix.

CI Can be compared with a random matrix ( $R I$ ), which is shown in Table 2.

Table 2: Values of the Random Index ( $R I)$

\begin{tabular}{llllllllllll}
\hline $\mathrm{n}$ & 1 & 2 & 3 & 4 & 5 & 6 & 7 & 8 & 9 & 10 & 11 \\
\hline$R I$ & 0 & 0 & 0.58 & 0.90 & 1.12 & 1.24 & 1.32 & 1.41 & 1.45 & 1.49 & 1.51 \\
\hline
\end{tabular}

The consistence radio (CR) can be calculated by formula (4).

$C R=\frac{C I}{R I}$

Where $C R<0.1$

\section{Result \&Analysis}

According to the data we have collected, we can get the results through the above steps.

Pairwise comparison matrix: 


$$
A=\left[\begin{array}{ccc}
1 & \frac{7}{5} & 7 \\
\frac{4}{7} & 1 & 4 \\
\frac{1}{7} & \frac{1}{4} & 1
\end{array}\right]
$$

Weight vector of criteria hierarchy.

$\omega=\left[\begin{array}{lll}0.5661 & 0.3471 & 0.0868\end{array}\right]^{T}$

In this hierarchy $C R=0.0135<0.1$.

Weight vector of alternatives hierarchy

Severity of epidemic: $\omega^{(1)}=\left[\begin{array}{lll}0.1322 & 0.3909 & 0.4769\end{array}\right]^{T}$

Per capital health expenditure: $\omega^{(2)}=\left[\begin{array}{lll}0.1791 & 0.2727 & 0.5481\end{array}\right]^{T}$

Medical staff per 10000 people: $\omega^{(3)}=\left[\begin{array}{lll}0.3010 & 0.3588 & 0.3402\end{array}\right]^{T}$

All of these weight vectors satisfy CR<0.1, then we can get a new vector $\omega^{\prime}=\left[\begin{array}{lll}\omega^{(1)} & \omega^{(2)} & \omega^{(3)}\end{array}\right]^{T}$

We get the combination weight vector $\omega_{f}$ by formula $\omega_{f}=\omega^{*} \omega^{\prime}$

$\omega_{f}=\left[\begin{array}{lll}0.1631 & 0.3471 & 0.4897\end{array}\right]$

At last, we can obtain medicine rights' weight in medicine distribution:

$\{$ Guinea, Liberia, SierraLeone $\}=\left\{\begin{array}{lll}0.1631 & 0.3471 & 0.4897\end{array}\right\}$

\section{Summary}

Taking into account multiple factors, we make the most of the medicine to reasonably distribute to these countries so as to save more patients by using AHP, where we take 3 indicators into consideration, which include Severity of Epidemic, Expenditure for Public Health and The Number of Medical Staff, finally, we get the weights of the three countries:

$\{$ Guinea, Liberia, SierraLeone $\}=\left\{\begin{array}{lll}0.1631 & 0.3471 & 0.4897\end{array}\right\}$

We will distribute medicines according to the weight of the three countries correspondingly, Sierra Leone gets most and Guinea gets least. Last but not least, our model can not only be used in Ebola epidemic, but also many other epidemics.

\section{References}

[1] file:///C:/Users/dell/Downloads/9781852337568-c1.pdf.

[2] http://www.dii.unisi.it/\%mocenni/Note_AHP.pdf. 